\title{
PARTIALLY CONTINUOUS DECOMPOSITIONS
}

\author{
R. H. BING
}

1. Introduction. In this paper we consider collections $G$ of mutually exclusive compact sets whose sum is a subset $X$ of a metric space. (We use the convention that a compact set is closed.) We shall be interested in whether or not $G$ is upper or lower semicontinuous. See $[8 ; 9]$ for treatments of such collections.

The collection $G$ is upper semicontinuous (or lower semicontinuous) if for each element $g$ of $G$ and each positive number $\epsilon$ there is a positive number $\delta$ such that if $g^{\prime}$ is an element of $G$ such that $\rho\left(g, g^{\prime}\right)<\delta$, then $g^{\prime} \subset V(g, \epsilon)$ (or $g \subset V\left(g^{\prime}, \epsilon\right)$ ). Here $\rho(A, B)$ denotes the distance between the sets $A$ and $B$ and $V(A, \epsilon)$ is the set of all points whose distance from $A$ is less than $\epsilon$.

If $\left\{g_{i}\right\}$ is a sequence of elements of $G$, the superior limit (or inferior limit) of $\left\{g_{i}\right\}$ is the set of all points $p$ such that each neighborhood of $p$ intersects infinitely many (or all but a finite number of the) elements of $\left\{g_{i}\right\}$. If these two limits are equal, they are called the limit of $\left\{g_{i}\right\}$, and $\left\{g_{i}\right\}$ is said to converge to this limit.

In the case where $X$ is compact, the collection $G$ is upper semicontinuous if for each converging sequence $\left\{p_{i}\right\}$ of points of $X$, the superior limit of $\left\{g_{i}\right\}$ ( $g_{i}$ contains $p_{i}$ ) lies in an element of $G$. The collection is lower semicontinuous if for each such sequence, each element that intersects the inferior limit of $\left\{g_{i}\right\}$ lies in it. The collection $G$ is continuous if it is both upper and lower semicontinuous.

The following scheme reminds us of the definitions of upper and lower semicontinuity as given in real variables.

$$
\begin{array}{ll}
\text { Lower semicontinuity } \leftrightarrow g_{0} \subset \text { limit }\left\{g_{i}\right\} . \\
\text { Upper semicontinuity } \leftrightarrow g_{0} \supset \text { limit }\left\{g_{i}\right\} . \\
\text { Continuity } \quad \leftrightarrow g_{0}=\text { limit }\left\{g_{i}\right\} .
\end{array}
$$

Here $\left\{g_{i}\right\}$ represents a converging sequence of elements of $G$ and $g_{0}$ is an element of $G$ that intersects the limit of this sequence.

If the elements of $G$ are connected, $G$ is called a monotone decomposition of $X$. An element (collection) is nondegenerate if it has more than one point (element). We show in Theorem 4 that if $G$ is a lower semicontinuous collection each of whose elements is a nondegenerate continuous curve and $X$ is a closed plane set, then $G$ is also upper semicontinuous.

Presented to the Society, April 30, 1954; received by the editors May 5, 1954. 
2. Decompositions and transformations. While upper semicontinuous decompositions have been widely studied, the notion of lower semicontinuous decompositions has not been used nearly so widely. We list some relationships between partially continuous decompositions and transformations. For the most part, these statements are well known for upper semicontinuous decompositions and are no more difficult to prove in the lower semicontinuous case.

Suppose $f$ is a transformation (not necessarily continuous) of a compact metric space $X$ onto a set $f(X)$ such that for each point $y$ of $f(X), f^{-1}(y)$ is closed. Then $f$ imposes a decomposition $G$ on $X-$ the elements of $G$ are the inverses of the points of $f(X)$. Here are two tests for the upper or lower semicontinuity of $G$ :

1. $G$ is upper semicontinuous if and only if, for each closed subset $A$ of $X, f^{-1} f(A)$ is closed.

2. $G$ is lower semicontinuous if and only if, for each open subset $D$ of $X, f^{-1} f(D)$ is open.

Now suppose $f(X)$ has a topology and $f$ is continuous. Then we have the following results:

3. $G$ is upper semicontinuous if $f(X)$ is metric (even Hausdorf).

4. $G$ is lower semicontinuous if $f(X)$ is $T_{1}$ and $f$ is open.

Conversely, suppose $G$ is a decomposition of $X$. Then there is a continuous transformation $g$ of $X$ determining $G$ and satisfying the following conditions.

5. $g(X)$ is metric if $G$ is upper semicontinuous.

6. $g(X)$ is $T_{1}$ and $g$ is open if $G$ is lower semicontinuous.

We may regard the elements of $G$ as the elements of the space $g(X)$. If $U$ is an open set in $X$, the collection of elements of $G$ which lie in $U$ is an open set in the metric space $g(X)$ of statement 5 ; the collection of elements of $X$ which intersect $U$ is an open set in the $T_{1}$ space $g(X)$ of statement 6 .

3. Reducing lower semicontinuous collections. Here we see how to reduce a locally compact continuum with a lower semicontinuous decomposition to a compact continuum with a decomposition which comes closer to being continuous. We define a continuum to be a closed connected set which is not necessarily compact.

If $g_{1}, g_{2}$ are two sets, the Hausdorff distance $H\left(g_{1}, g_{2}\right)$ between them is the least upper bound of $\rho\left(x, g_{i}\right) ; i=1,2 ; x \in g_{1}+g_{2}$.

Theorem 1. Suppose $G$ is a monotone lower semicontinuous decomposition of a locally compact continuum $X$ and $G$ has more than one element. For each element $g_{0}$ of $G$ and each positive number $\epsilon$ there is a compact continuum $M$ such that $M$ is the sum of elements of $G, M$ prop- 
erly contains $g_{0}$, and the Hausdorff distance between each pair of elements of $G$ in $M$ is less than $\epsilon$.

Proof. Regard $X$ as all of space. Since a locally compact connected metric space is perfectly separable, there is a countable basis for $X$ that is, a countable collection $\left\{D_{i}\right\}$ of open sets such that each open set in $X$ is the sum of elements of $\left\{D_{i}\right\}$.

If there is a continuum in $X-D_{1}$ that properly contains $g_{0}$ and which is the sum of elements of $G$, let $M_{1}$ be such a continuum; otherwise $M_{1}=X$. If there is a continuum in $M_{1}-D_{2}$ which properly contains $g_{0}$ and is the sum of elements of $G$, let $M_{2}$ be such a continuum; otherwise $M_{2}=M_{1}$. In general, $M_{i+1}$ is a subcontinuum of $M_{i}$ which properly contains $g_{0}$ and is the sum of elements of $G$; it misses $D_{i+1}$ if this is possible; otherwise $M_{i+1}=M_{i}$.

The intersection of $M_{1}, M_{2}, \cdots$ is a closed set $M^{\prime}$ that contains $g_{0}$ and is the sum of elements of $G$. We show that $M^{\prime}=g_{0}$.

Let $N(\epsilon)$ be the sum of all elements of $G$ in $M^{\prime}$ whose distance (not Hausdorff distance) from $g_{0}$ is greater than or equal to $\epsilon$. It follows from the lower semicontinuity of $G$ that $N(\boldsymbol{\epsilon})$ is closed. Since $M^{\prime}=g_{0}$ $+N(1)+N(1 / 2)+N(1 / 3)+\cdots$, it follows from the Baire category theorem that unless $g_{0}=M^{\prime}$, there is an integer $j$ such that $N(1 / j)$ contains a nonnull subset $E$ of $M^{\prime}$ open relative to $M^{\prime}$.

Assume $g_{0}$ is not a component of $M^{\prime}$. Let $R$ be a continuum in $M^{\prime}-N(1 / j)$ that properly contains $g_{0}$ and $M^{\prime \prime}$ be the closure of the sum of all elements of $G$ that intersect $R$. Then $M^{\prime \prime}$ does not intersect $E$, but properly contains $g_{0}$, and is the sum of elements of $G$. If $p$ is a point of $E$, there is an integer $r$ such that $D_{r}$ contains $p$ and $D_{r} \cdot M^{\prime}$ lies in $E$. But $M_{r}$ would not contain $p$ because $M^{\prime \prime}$ is a continuum in $M^{\prime}-D_{r}$ (hence in $M_{r-1}-D_{r}$ ) which properly contains $g_{0}$ and is the sum of elements of $G$. Hence, the assumption that $g_{0}$ is not a component of $M^{\prime}$ has led to the contradiction that the point $p$ of $M^{\prime}$ does not belong to $M^{\prime}$.

Since $g_{0}$ is compact, lies in an open set $D$ whose closure is compact, and is a component of the intersection of the decreasing sequence $M_{1}, M_{2}, \cdots$ of continua, $g_{0}$ is this intersection.

Since $G$ is lower semicontinuous there is a positive number $\delta$ such that if $g$ is an element of $G$ such that $\rho\left(g, g_{0}\right)<\delta$, then for each point $p$ of $g_{0}, \rho(p, g)<\epsilon / 2$. Since $g_{0}$ is the intersection of the continua of $M_{1}, M_{2}, \cdots$ and lies in an open set whose closure is compact, there is an integer $k$ such that $M_{k}$ is compact and for each point $p$ of $M_{k}$, $\rho\left(p, g_{0}\right)$ is less than either $\epsilon / 2$ or $\delta$. We can let $M=M_{k}$ because if $g$ is an element of $G$ in $M_{k}$, then $H\left(g, g_{0}\right)<\epsilon / 2$.

We note that the preceding theorem is not true if we replace lower 
semicontinuity by upper semicontinuity. The theorem implies that if $g_{0}$ is an element of $G$ there is a sequence of different elements of $G$ converging to $g_{0}$.

Consider two examples of lower semicontinuous decompositions.

EXAmple 1. We describe a lower semicontinuous decomposition $G_{\epsilon}$ of a unit cube into arcs and disks. The decomposition is not upper semicontinuous.

Consider a base $B$ of this cube. If $S$ is a slice of the cube parallel to $B$ and at an irrational distance from $B, S$ is an element of $G_{\epsilon}$.

If $S$ is a slice of the cube at a distance $p / q$ (in lowest terms) from $B$, there is a continuous decomposition $G(S, \epsilon / q)$ of $S$ such that each element of $G(S, \epsilon / q)$ is an arc which comes within $\epsilon / q$ of each point of $S$. Each element of $G(S, \epsilon / q)$ is an element of $G$.

ExAmple 2. We may be unable to get $M$ for Theorem 1 on which $G$ is continuous. We give here an example of a lower semicontinuous decomposition $G$ of a Hilbert cube such that if $G^{\prime}$ is any infinite subcollection of $G$ whose sum is a continuum, $G^{\prime}$ fails to be upper semicontinuous.

We may regard the Hilbert cube as the cartesian product $C_{1}$ $\times C_{2} \times C_{3} \times \cdots$ where $C_{i}$ is a unit cube. Frequently a Hilbert cube is considered as the cartesian product of closed intervals $I_{n}$ where $I_{n}$ $=[-1 / n, 1 / n]$ but by adjusting the metric we may suppose $I_{n}$ $=[0,1]$. Similarly, we may choose a metric for $C_{1} \times C_{2} \times C_{3} \cdots$ to give it the familiar metric for the Hilbert cube. For each positive number $\epsilon$ and each cube $C_{i}$ let $G\left(\epsilon, C_{i}\right)$ be a particular decomposition of $C_{i}$ into disks and arcs as described in Example 1. There is a countable collection $W\left(\epsilon, C_{i}\right)=\left\{w_{j}\right\}$ of arcs in $G\left(\epsilon, C_{i}\right)$ such that each continuum in $C_{i}$ which is the sum of infinitely many elements of $G\left(\epsilon, C_{i}\right)$ contains some element of $W\left(\epsilon, C_{i}\right)$. Let $G^{\prime}\left(\epsilon, C_{i}\right)=G\left(\epsilon, C_{i}\right)$ $-W\left(\epsilon, C_{i}\right)$.

If $g^{1} \in G^{\prime}\left(1, C_{1}\right), g^{1} \times C_{2} \times C_{3} \times \cdots$ is an element of $G$. If $w_{i}^{1}$ $\in W\left(1, C_{1}\right)$, consider $G\left(\epsilon_{i}^{1}, C_{2}\right)$ where $\epsilon_{i}^{1}=1 / i q$ and $w_{i}^{1}$ is in the slice of $C_{1}$ at a distance of $p / q$ from the base of $C_{1}$.

If $g^{2} \in G^{\prime}\left(\epsilon_{i}^{1}, C_{2}\right), w_{1}^{1} \times g^{2} \times C_{3} \times \cdots$ is an element of $G$. If $w_{j}^{2}$ $\in W\left(\epsilon_{i}^{1}, C_{2}\right)$, consider $G\left(\epsilon_{j}^{2}, C_{3}\right)$ where $\epsilon_{j}^{2}=\epsilon_{i}^{1} / j q$ and $w_{j}^{2}$ is in the slice of $C_{2}$ at a distance of $p / q$ from the base of $C_{2}$. If $g^{3} \in G^{\prime}\left(\epsilon_{j}^{2}, C_{3}\right), w_{i}^{1} \times w_{j}^{2}$ $\times g^{3} \times C_{4} \times \cdots$ is an element of $G$.

The process is continued. An element of $G$ is either of the form

$$
\begin{aligned}
& w_{n_{1}}^{1} \times w_{n_{2}}^{2} \times w_{n_{3}}^{8} \times \cdots, g^{1} \times C_{2} \times C_{3} \times \cdots, \text { or } \\
& w_{n_{1}}^{1} \times w_{n_{2}}^{2} \times \cdots \times w_{n_{i}}^{i} \times g^{i+1} \times C_{i+2} \times \cdots
\end{aligned}
$$


where $w_{n}^{i} \in W\left(\epsilon_{n_{i-1}}^{i-1}, C_{i}\right), g^{i} \in G^{\prime}\left(\epsilon_{n_{i-1}}^{i-1}, C_{i}\right), \epsilon_{n_{i}}^{i}=\epsilon_{n_{i-1}}^{i-1} / n_{i} q$, and $w_{n_{i}}^{i}$ is in the slice of $C_{i}$ at a distance of $p / q$ from the base of $C_{i}$.

Suppose $M^{\prime}$ is a continuum in the Hilbert cube which is the sum of a subcollection $G^{\prime}$ of $G$. First we show that if $M^{\prime}$ intersects each of $S^{\prime} \times C_{2} \times C_{3} \times \cdots$ and $S^{\prime \prime} \times C_{2} \times C_{3} \times \cdots$ where $S^{\prime}$ and $S^{\prime \prime}$ are different slices of $C_{1}$ parallel to its base, then $G^{\prime}$ is not upper semicontinuous. There is a sequence of slices $S_{1}, S_{2}, \ldots$ each at an irrational distance from the base of $C_{1}$ converging to a slice $S_{0}$ at a rational distance from this base and such that each $S_{i}$ separates $S^{\prime}$ from $S^{\prime \prime}$. Then $G^{\prime}$ is not upper semicontinuous at any of its elements in $S_{0} \times C_{2} \times C_{3} \times \cdots$.

Suppose $M^{\prime}$ lies in $S \times C_{2} \times C_{3} \times \cdots$ but intersects two elements of $G\left(1, C_{1}\right)$ in the slice $S$. Then there is a sequence $g_{1}, g_{2}, \cdots$ of elements of $G^{\prime}\left(1, C_{1}\right)$ in this slice converging to an element $w$ of $W\left(1, C_{1}\right)$ in this slice and such that $M^{\prime}$ intersects and contains each $g_{i} \times C_{2} \times C_{3}$ $\times \ldots$ But $G^{\prime}$ is not upper semicontinuous at any element in $w \times C_{2} \times C_{3} \times \cdots$.

We have found that if $G^{\prime}$ is continuous, the first coordinates of any two elements of $G^{\prime}$ are the same. Here $w_{n_{i}}^{i}, g^{i}$, and $C_{i}$ are regarded as coordinates of an element of $G^{\prime}$. A similar argument shows that the other coordinates are also equal. Hence, $G^{\prime}$ has only one element.

Question. For some continua $M$ (for an arc but not for a Hilbert cube) the following statement is true: For each nondegenerate monotone lower semicontinuous decomposition $G$ of $M$ there is a subcontinuum $M^{\prime}$ of $M$ which is the sum of an infinite subcollection $G^{\prime}$ of $G$ such that $G^{\prime}$ is continuous. It would be interesting to know for what types of continua this statement is true. The statement with "lower" replaced by "upper" is not true for any nondegenerate compact continuum.

If we had not been interested in getting a connected $M$, we would not have needed to suppose that $G$ is monotone. We would have been able to conclude that the collection of elements of $G$ in $M$ is continuous. Consider the following result.

TheOREM 2. Suppose G has uncountably many elements and is a lower semicontinuous decomposition of a locally compact separable metric space $X$. There is an uncountable subcollection $G^{\prime}$ of $G$ such that $G^{\prime}$ is continuous and the sum of the elements of $G^{\prime}$ is compact.

Proof. Since $X$ has a countable covering $\left\{D_{i}\right\}$ by open sets with compact closures and each element of $G$ is covered by. a finite subcollection of $\left\{D_{i}\right\}$, there is a finite subcollection of $\left\{D_{i}\right\}$ that covers uncountably many elements of $G$. Hence, with no loss of generality 
we suppose that $X$ is compact.

The methods of Theorem 1 reveal that there is a compact set $M_{0}$ which is the sum of uncountably many elements of $G$ such that if $g_{1}, g_{2}$ are two elements of $G$ in $M_{0}$, then $H\left(g_{1}, g_{2}\right)<1$.

Again these methods reveal that $M_{0}$ contains two mutually exclusive compact subsets $C_{1}^{1}, C_{2}^{1}$ such that each is the sum of uncountably many elements of $G$ and such that if $g_{1}, g_{2}$ are elements of $G$ in $C_{i}$ $(i=1,2)$, then $H\left(g_{1}, g_{2}\right)<1 / 2$. Let $M_{1}=C_{1}^{1}+C_{2}^{1}$.

In general, $M_{i}$ is the sum of $2^{i}$ mutually exclusive compact sets $C_{1}^{i}, C_{2}^{i}, \cdots, C_{2 i}^{\mathbf{s}}$ such that $C_{j}^{i}$ is the sum of uncountably many elements of $G$ and if $g_{1}, g_{2}$ are two elements of $G$ in $C_{j}^{i}, H\left(g_{1}, g_{2}\right)<1 / 2^{i}$. Each $C_{j}^{t}$ contains two of the compact sets $C_{r}^{t+1}, C_{s}^{t+1}$ in $M_{i+1}$.

The collection $G^{\prime}$ of elements of $G$ in $M^{\prime}=M_{0} \cdot M_{1} \cdots$ is continuous. Also, $G^{\prime}$ has uncountably many elements since it is a Cantor set with respect to its elements as points.

Although this concludes the proof of Theorem 2, it is of interest that if $X$ is compact, and $N(\epsilon)$ is the sum of the elements $g$ of $G$ at which $G$ fails to be upper semicontinuous by $\epsilon$ (for each $i$ there is a $g_{i}$ in $G$ such that $\left.\rho\left(g, g_{i}\right)<1 / i, g_{i} \subset V(g, \epsilon)\right)$, then $N(\boldsymbol{\epsilon})$ is closed and contains no open set. Hence the sum of the elements of $G$ at which $G$ is continuous is a dense $G_{\delta}$ set.

In a bicompact connected $T_{1}$ space it does not follow that for each pair of points there is a continuum irreducible between them. Hence, Theorem 3 shows that not each bicompact $T_{1}$ space is a space $g(X)$ of the type referred to in statement 6 of the preceding section Transformations and decompositions. The following result follows from the same type of argument given in the proof of Theorem 1.

THEOREM 3. If $G$ is a monotone lower semicontinuous decomposition of a connected compact metric space $X$ and $A, B$ are two nonnull closed subsets of $X$, there is a continuum $M$ in $X$ which is irreducible with respect to being a continuum which is the sum of elements of $G$ and intersects each of $A, B$.

If $M_{A}$ and $M_{B}$ denote the sum of the elements of $G$ in $M$ which intersect $A$ and $B$ respectively, then each of $M-M_{A}, M-M_{B}$, and $M$ $-\left(M_{A}+M_{B}\right)$ is connected.

4. Decompositions whose elements are continuous curves. In this section we shall be interested in a lower semicontinuous decomposition $G$ of a closed planar set such that the elements of $G$ are nondegenerate continuous curves. (A continuous curve is a compact locally connected continuum.) We find that $G$ must be upper semicontinuous also. Example 1 shows that the hypothesis of Theorem 4 
must include the condition that the sum of the elements of $G$ is planar.

TheOREM 4. Suppose $G$ is a lower semicontinuous decomposition of a closed planar set such that each element of $G$ is a nondegenerate continuous curve. Then $G$ is also upper semicontinuous.

Proof. Suppose $E_{1}, E_{2}, E_{3}$ are three point sets. We say that the arc $\alpha$ is hooked with respect to $E_{1}, E_{2}, E_{3}$ if it contains two $\operatorname{arcs} \alpha_{1}, \alpha_{2}$ with at most an end point in common such that $\alpha_{1}$ intersects all three of $E_{1}, E_{2}, E_{3}$ and $\alpha_{2}$ intersects two of them. Compare this notion of being hooked with the idea of a sequence of chains being hooked as given by Anderson on page 650 of [1].

On the assumption that $G$ is not upper semicontinuous we show that there is a decreasing sequence of compact continua $M_{1}, M_{2}, \ldots$ and three decreasing sequences of domains $D_{i 1}, D_{i 2}, \cdots(i=1,2,3)$ such that:

(a) $M_{j}$ is the sum of uncountably many elements of $G$.

(b) $M_{1} \cdot M_{2} \cdot \ldots$ is an element $\alpha_{0}$ of $G$ which is an arc.

(c) Each element of $G$ in $M_{j}$ which is an arc is hooked with respect to $D_{1 j}, D_{2 j}, D_{3 j}$.

(d) $\bar{D}_{i j+1} \subset D_{i j}$ and $D_{i 1} \cdot D_{i 2} \ldots$ is a point $p_{i}$ with $p_{1} \neq p_{2} \neq p_{3} \neq p_{1}$.

But since the arc $\alpha_{0}$ cannot be hooked with respect to the three points $p_{1}, p_{2}, p_{3}$ there is an integer $j$ such that $\alpha_{0}$ is not hooked with respect to $D_{1 j}, D_{2 j}, D_{3 j}$. Hence the assumption that $G$ is not upper semicontinuous will have led us to a contradiction.

Suppose $g$ is an element of $G$ at which $G$ is not upper semicontinuous. Then there is a sequence of elements $g_{1}, g_{2}, \cdots$ converging to a closed set $W$ which properly contains $g$. From Theorem 1 there is a compact continuum $M$ in $W$ which properly contains $g$, is the sum of elements of $G$, and is such that if $g^{\prime}, g^{\prime \prime}$ are two elements of $G$ in $M$, then the Hausdorff distance $H\left(g^{\prime}, g^{\prime \prime}\right)<1$.

Let $G_{M}$ be the collection of elements of $G$ in $M$. We now establish two properties of $G_{M}$ that we shall use.

Property 1. All but a countable number of elements of $G_{M}$ are arcs. Since the plane does not contain uncountably many triods, all but a countable number of elements of $G_{M}$ are arcs or simple closed curves. If $G_{M}$ contained uncountably many simple closed curves, it would contain three such that some one of the three separated the other two from each other in the plane. This is impossible for the sum of the three simple closed curves would belong to the inferior limit of a sequence of plane continua which does not intersect the sum.

Property 2. $G_{M}$ is not upper semicontinuous. If $G_{M}$ were upper 
semicontinuous, it would not contain infinitely many elements of the sequence $g_{1}, g_{2}, \cdots$. Anderson has shown [1] that if $G^{\prime}$ is a continuous collection of nondegenerate continuous curves filling a compact continuum $M^{\prime}$, then $M^{\prime}$ contains the interior of a circle. But $M$ cannot contain the interior of a circle since it is a subset of the inferior limit of the sequence $g_{1}, g_{2}, \ldots$ which has infinitely many elements which do not intersect $M$. Hence, $G_{M}$ is not upper semicontinuous.

We note that if $M^{\prime}$ is any subcontinuum of $M$ which is the sum of uncountably many elements of $G$, then the collection of elements of $G$ in $M^{\prime}$ has properties 1 and 2.

Let $\alpha$ be an arc in $M$ which is an element of $G$, and $D_{11}, D_{21}, D_{31}$ be three mutually exclusive domains intersecting $\alpha$. We show that there is a continuum $M_{1}$ in $M$ which is the sum of uncountably many elements of $G$ and such that each arc in $M_{1}$ which is an element of $G$ is hooked with respect to $D_{11}, D_{21}, D_{31}$.

Let $D_{1}, D_{2}, \cdots, D_{n}$ be a chain of open topological disks covering $\alpha$ such that $D_{i} \cdot D_{j}$ is connected or null according as $i$ and $j$ are or are not adjacent and each of $D_{11}, D_{21}, D_{31}$ contains a link of the chain which intersects $\alpha$. We suppose that $D_{n_{i}}$ intersects $\alpha, D_{n_{i}} \subset D_{i 1}$, and $n_{1}<n_{2}<n_{3}$. For convenience we suppose that $D_{n_{1}}, D_{n_{2}}, D_{n_{3}}$ are the interiors of circles with centers on the $x$-axis and that if $L$ is the part of the $x$-axis to the left of $D_{n_{1}}$ and $R$ is the part to the right of $D_{n_{3}}$, then $L+R$ does not intersect any link of the chain.

By Theorem 1 there is a continuum $M^{\prime}$ in $M$ such that $M^{\prime}$ is covered by $D_{1}, D_{2}, \cdots, D_{n}, M^{\prime}$ contains $\alpha$, and $M^{\prime}$ is the sum of an uncountable subcollection $G^{\prime}$ of $G$ such that each element of $G^{\prime}$ will intersect each of $D_{n_{1}}, D_{n_{2}}$. Each element will also intersect $D_{n_{2}}$ because $D_{n_{3}}$ is between $D_{n_{1}}$ and $D_{n_{3}}$.

In each element $g$ of $G^{\prime}$, select an arc $h_{g}$ which is irreducible from $\bar{D}_{n_{1}}$ to $\bar{D}_{n_{3}}$ and denote the collection of all such arcs by $H$. The elements of $H$ have a natural linear order-for example, $h^{\prime}$ is above $h^{\prime \prime}$ if it lies in the complementary domain of $L+D_{n_{1}}+h^{\prime \prime}+D_{n_{2}}+R$ which is unbounded from above.

Since $G^{\prime}$ is not upper semicontinuous, there is a converging sequence $g_{2}^{\prime}, g_{3}^{\prime}, \cdots$ of elements of $G^{\prime}$ and two elements $g_{0}^{\prime}, g_{1}^{\prime}$ of $G^{\prime}$ which belong to the inferior limit of $g_{2}^{\prime}, g_{3}^{\prime}, \ldots$. Let $h_{i}$ be the element of $H$ in $g_{i}^{\prime}$. Since some subsequence of $h_{2}, h_{3}, \cdots$ is monotone, we suppose that $h_{i+1}$ is above $h_{i}$ if $i>1$.

In the linear order of $H$, one of the sets $h_{0}, h_{1}$ is separated from all but a finite number of elements of $h_{2}, h_{3}, \cdots$ by some element of $h_{0}, h_{1}, h_{2}, \cdots$. For convenience we suppose that $h_{j}$ separates $h_{0}$ from each element of $h_{r}, h_{r+1}, \cdots$. 
Let $N$ be a connected neighborhood in $D_{n_{2}}$ which contains a point of $h_{0}$ but no point of $h_{j}$. Since $G^{\prime}$ is lower semicontinuous, some element $g_{k}^{\prime}$ of $g_{r}^{\prime}, g_{r+1}^{\prime}, \cdots$ intersects $N$. Then the component of $g_{k}^{\prime}-\left(D_{n_{1}}+D_{n_{3}}\right)$ that contains $h_{k}$ also intersects $\bar{D}_{n_{1}}, D_{n_{2}}$, and $\bar{D}_{n_{3}}$ but not $N$, while another component of it intersects $N$. Now if $M_{1}$ is a continuum sufficiently close to $g_{k}^{\prime}$ (and there is one sufficiently close by Theorem 1) and the sum of uncountably many elements of $G^{\prime}$, then each element of $G^{\prime}$ in $M_{1}$ which is an arc is hooked with respect to $D_{n_{1}}, D_{n_{2}}$, and $D_{n_{3}}$ and hence with respect to $D_{11}, D_{21}, D_{31}$.

Now that we have obtained $D_{11}, D_{21}, D_{31}$, and $M_{1}$, all we need is an iteration of the preceding argument to get the three sequences $D_{i 1}, D_{i 2}, \cdots(i=1,2,3)$ and the sequence $M_{1}, M_{2}, \cdots$. Once $M_{n}$, $D_{1 n}, D_{2 n}, D_{3 n}$ are found, we consider an arc $\alpha$ in $M_{n}$ which is an element of $G$. Let $D_{j n+1}(j=1,2,3)$ be an open set of diameter less than $1 / n$ which intersects $\alpha$ and whose closure lies in $D_{j n}$. We use a $1 / n$-chain covering $\alpha$ and show the existence of $M_{n+1}$ by the same methods used to show the existence of $M_{1}$. The reason that condition (b) is satisfied is that an arc is the only nondegenerate snake-like continuous curve. (A continuum is snake-like [4] if for each positive number $\epsilon$ it can be covered by an $\epsilon$-chain.)

Theorem 4 does not hold if we omit some of the conditions in the hypothesis. We cite some examples to illustrate this.

EXAMPLE 3. If the requirement were not made that the elements of $G$ be nondegenerate, we could have let $G$ be the collection of points on the $y$-axis from $(0,0)$ to $(0,1)$ and the collection of vertical intervals of length 1 with lower ends on the sect $(a, b]$ where $a=(0,0)$ and $b=(1,0)$. Then $G$ is lower semicontinuous but not upper semicontinuous.

EXAmple 4. It is essential to suppose that the elements of $G$ be continuous curves and not just continua. Let $M$ be a nondegenerate hereditarily indecomposable continuum. Such continua have been described by Knaster [6], Moise [7], and Bing [2;3]. Kelley has shown [5] that there is a continuous decomposition $G^{\prime}$ of such a continuum into nondegenerate continua. Let $g$ be an element of $G^{\prime}$ and $G^{\prime \prime}$ be a continuous decomposition of $g$ into nondegenerate continua. Then the collection consisting of elements of $G^{\prime \prime}$ and elements of $G^{\prime}$ other than $g$ is lower semicontinuous but not upper semicontinuous.

\section{REFERENCES}

1. R. D. Anderson, Continuous collections of continuous curves in the plane, Proc. Amer. Math. Soc. vol. 3 (1952) pp. 647-657.

2. R. H. Bing, Concerning hereditarily indecomposable continua, Pacific Journal of Mathematics vol. 1 (1951) pp. 43-51. 
3. - Higher-dimensional hereditarily indecomposable continua, Trans. Amer. Math. Soc. vol. 71 (1951) pp. 267-273.

4. - Snake-like continua, Duke Math. J. vol. 18 (1951) pp. 653-663.

5. J. L. Kelley, The hyperspaces of a continuum, Trans. Amer. Math. Soc. vol. 52 (1942) pp. 22-36.

6. B. Knaster, Un continu dont tout sous-continu est indécomposable, Fund. Math. vol. 3 (1922) pp. 247-286.

7. E. E. Moise, An indecomposable plane continuum which is homeomorphic to each of its nondegenerate subcontinua, Trans. Amer. Math. Soc. vol. 63 (1948) pp. 581-594.

8. R. L. Moore, Foundations of point set theory, Amer. Math. Soc. Colloquium Publications, vol. 13, New York, 1932.

9. G. T. Whyburn, Analytic topology, Amer. Math. Soc. Colloquium Publications, vol. 28, New York, 1942.

UNIVERSITY OF WISCONSIN

\section{UNE PROPRIETE TOPOLOGIQUE DES DOMAINES DE RUNGE}

\section{JEAN-PIERRE SERRE}

Nous dirons qu'un domaine $X$ de l'espace numérique complexe $\boldsymbol{C}^{n}$ est un domaine de Runge si:

1. $X$ est un domaine d'holomorphie.

2. Toute fonction holomorphe sur $X$ est limite uniforme sur tout compact de polynômes.

On sait que, si $n=1, X$ est simplement connexe. Nous allons généraliser ce résultat:

ThÉORÈme. Le $n^{\text {eme }}$ nombre de Betti d'un domaine de Runge de $\mathrm{C}^{n}$ est nul.

Démonstration. Soit $C^{n}(X)$ l'espace vectoriel des formes différentielles $\omega=f\left(z_{1}, \cdots, z_{n}\right) d z_{1} \wedge \cdots \wedge d z_{n}$, où $f$ est holomorphe sur $X$. Une telle forme est toujours fermée, i.e. $d \omega=0$. Soit $B^{n}(X)$ le sous-espace de $C^{n}(X)$ formé des éléments $\omega$ qui sont de la forme $d \alpha$, où $\alpha$ est une forme différentielle holomorphe de degré $n-1$. D'après le Théorème 1 de [2], qui s'applique à cause de l'hypothèse 1 , l'espace quotient $C^{n}(X) / B^{n}(X)$ est isomorphe à $H^{n}(X, C)$, et tout revient donc à montrer que $B^{n}(X)=C^{n}(X)$.

Munissons $C^{n}(X)$ de la topologie de la convergence compacte. Alors:

(a) $B^{n}(X)$ est fermé dans $C^{n}(X)$.

Received by the editors April 24, 1954. 